\title{
Remodelación, Transformación y Rehabilitación. Tres formas de intervenir en la Vivienda Social del siglo xx
}

\author{
Urban Remodeling, Transformation and Renovation. \\ Three ways of intervening on twentieth century Social Housing \\ N. Cervero Sánchez ${ }^{(*)}$, L. Agustín Hernández ${ }^{(*)}$
}

\begin{abstract}
RESUMEN
La vivienda social construida en España entre 1939 y 1976 supone un patrón urbano extendido en la ciudad contemporánea. Sus condiciones de partida y el efecto del tiempo la han llevado a desarrollar una serie de obsolescencias, que hacen necesario intervenir para conseguir su revitalización. Los programas desarrollados en los últimos años se ocupan de dotar a las edificaciones de unos niveles de habitabilidad adecuados, pero también de su articulación como grupo, y de su integración en la distribución socioeconómica del núcleo urbano. Mediante el análisis de la Remodelación Urbana de Orcasitas (Madrid) y de las Viviendas del Gobernador (Barcelona), la Transformación Urbana de La Mina (Barcelona) y la Rehabilitación Integral del Barrio de Lourdes (Tudela) se estudiará la evolución de estos modelos de actuación.
\end{abstract}

Palabras clave: Regeneración urbana; rehabilitación; remodelación; vivienda social.

\begin{abstract}
Social housing built in Spain between 1939 and 1976 constitutes a widespread urban pattern in contemporary cities. Its initial conditions, along with the effects of time, have led to obsolescence, requiring refurbishment for revitalisation. Programs developed over the last few years not only have provided the buildings with the appropriate standards of living conditions, but also have articulated them as a whole, integrating them into the socio-economic configuration of their urban area. The evolution of these intervention models will be reviewed analyzing the Urban Remodeling of Orcasitas (Madrid) and Viviendas del Gobernador housing (Barcelona), the Urban Transformation of La Mina (Barcelona) and the Integral Renovation of Barrio de Lourdes (Tudela).
\end{abstract}

Keywords: Urban regeneration; rehabilitation; remodeling; social housing.

(*) Universidad de Zaragoza. Zaragoza (España).

Persona de contacto/Corresponding author: ncervero@unizar.es (N. Cervero Sánchez)

Cómo citar este artículo/Citation: Cervero Sánchez, N., Agustín Hernández, L. (2015). Remodelación, Transformación y Rehabilitación. Tres formas de intervenir en la Vivienda Social del siglo xx. Informes de la Construcción, 67(EXTRA-1): mo26, doi: http://dx.doi.org/10.3989/ic.14.049.

Licencia/License: Salvo indicación contraria, todos los contenidos de la edición electrónica de Informes de la Construcción se distribuyen bajo una licencia de uso y distribución Creative Commons Reconocimiento no Comercial 3.o. España (cc-by-nc). 


\section{INTRODUCCIÓN}

Para garantizar el derecho a una vivienda digna, recogido en la Constitución (art. 47), los poderes públicos intervienen en el mercado inmobiliario, facilitando las llamadas viviendas protegidas, destinadas a población con recursos insuficientes para acceder al mercado libre. Estas actuaciones comenzaron a ser reguladas en 1911 con la Ley de Casas Baratas, y se convierten en extensivas en las décadas posteriores a la Guerra Civil. En concreto los modelos estudiados se construyen desde 1939 hasta 1976, cuando comienza el Tercer Plan Nacional de Vivienda. Por sus condiciones iniciales y el desgaste temporal, han desarrollado una serie de obsolescencias que se manifiestan en diferentes ámbitos: constructivo, social y de integración en la ciudad. Por ello, desde la administración se está procediendo con actuaciones de gran complejidad.

En términos generales, en función de los problemas y tipos de gestión, encontramos en la política de recuperación residencial tres fórmulas de intervención:

- Remodelación Urbana: consiste en la sustitución de la edificación atendiendo a una nueva ordenación urbanística.

- Transformación Urbana: es una intervención híbrida, en la que se combinan distintos niveles de actuación que pueden variar desde la remodelación a la rehabilitación ligera.

- Rehabilitación Integral: implica la mejora de la edificación y del espacio público, con el fin de actualizar sus condiciones de habitabilidad a los requerimientos actuales.

Se han seleccionado ejemplos representativos de cada uno de estos niveles de intervención que se complementan entre sí, para presentar un análisis crítico del panorama actual en este tipo de operaciones. Para su explicación, se han ordenado temática y cronológicamente: la Remodelación Urbana del Poblado Dirigido de Orcasitas en Madrid y de las Viviendas del Gobernador en Barcelona, la Transformación Urbana del barrio de La Mina en Barcelona, y la Rehabilitación Integral del barrio de Lourdes de Tudela en Navarra.

\section{REMODELACIÓN URBANA}

Las primeras remodelaciones que se llevan a cabo manifiestan la falta de un plan generador de estrategia territorial, que posibilite la incorporación urbana o socioeconómica de los ámbitos tratados. La remodelación del Poblado Dirigido de Orcasitas (1976-1986) marca el comienzo del Plan de Remodelación de Barrios de Madrid, un proceso que transforma la periferia más degradada, durante los años 1979 al 1996. Se trata de una operación a gran escala, 837,8 ha, que consiste en la regeneración de 30 barrios de infravivienda y promoción oficial, mediante la construcción de 39.000 viviendas, infraestructuras y equipamientos, y que alcanza una inversión total de aproximadamente 1.860 millones de euros (1).

El Poblado Dirigido de Orcasitas, es un conjunto de 40,27 ha y planta prácticamente cuadrada proyectado por R. Leoz de la Fuente y J. Ruiz Hervás, y construido entre los años 1957 y 1965. Ordenado con una estructura en retícula, lo define un eje principal y la edificación abierta, dispuesta en bandas paralelas y perpendiculares a las vías. En cuanto a la morfología edificatoria, existen dos tipologías, una en superficie, con 720 viviendas unifamiliares en hilera, y otra en altura, con bloques lineales de doble crujía y seis plantas, hasta completar un total de 2.964 viviendas. Los servicios comunitarios, en proyecto se ubican en el perímetro, pero la mayoría no llegan a construirse.

El problema del poblado es originado por la naturaleza del suelo en el que se asienta, compuesto por arcillas expansivas. Pocos meses después de terminar la ejecución de la estructura del poblado, aparecen las primeras grietas y en 1961 son ya un asunto que preocupa a los técnicos y a los futuros vecinos (2). A pesar de las medidas tomadas desde el primer momento, el hecho de que el terreno sufra un proceso gradual de desecación provoca asientos, que lejos de estabilizarse, se agravan hasta mermar la habitabilidad, incluso la seguridad de las edificaciones. Esta situación crítica, provoca que en 1975, diez años después de su construcción, comience a trasladarse población fuera del poblado, lo que genera uno de los conflictos sociales más importantes del momento. Con el Decreto 323/1976 de actuaciones urgentes del Instituto Nacional de la Vivienda en el Poblado Dirigido de Orcasitas, se pone en marcha el Plan Parcial para la remodelación del barrio. Pero no se consolida hasta que se dicta la Orden Comunicada en $1979^{1}$, que contempla por primera vez este tipo de procesos en la ciudad de Madrid. La intervención en Orcasitas, dirigida por M. Gutiérrez Plaza y J.I. Casanova Fernández, junto con E. Hernández Fernández como asesor técnico de la Asociación de Vecinos, se convierte en pionera del Plan. La fuerza del movimiento vecinal continúa durante una operación, en la que los técnicos se pliegan a las decisiones de los usuarios, que definen los criterios de la nueva ordenación, intervienen en las decisiones del proyecto y supervisan la obra, en un tipo de colaboración singular.

La sustitución de la edificación se realiza en tres fases escalonadas, permitiendo el realojo de una sobre la anterior. El resultado responde a una ordenación en la que se repone el mismo número de viviendas del barrio original manteniendo una densidad de 73,60 viviendas por ha, y en la que los espacios libres, viarios y aparcamientos corresponden prácticamente a un $80 \%$ de la superficie total (3) (Figura 1.1). Por razones de urgencia, se mantiene la calificación de edificación abierta y por decisión popular, se reponen las dos tipologías previas, cuestiones que condicionan totalmente el resultado de la operación. Se conserva en esencia la estructura urbana anterior, manteniendo el eje Norte-Sur central y otro lateral de acceso rodado y vías secundarias con fondos de saco para aparcamientos en superficie. En el interior de las manzanas, la convivencia de las dos tipologías previas da lugar a espacios públicos contrapuestos: las viviendas unifamiliares se disponen sobre una trama saturada, y los bloques, que crecen a las once alturas y liberan la planta baja, conforman entre ellos grandes plazas sin uso definido. Las características dimensionales de estos espacios, su tratamiento, y su falta de función llegan a eliminar su sentido, evitando conexión alguna con la trama viaria. Por otra parte, las funciones aparecen fuertemente sectorizadas, con equipamientos concentrados en el extremo norte, un área calificada como parque, cons-

\footnotetext{
Este primer marco jurídico se ve fortalecido en 1984 con el Real Decreto 1133/1984 y con la transferencia de competencias a la Comunidad Autónoma, que completa el proceso a través del Instituto de la Vivienda de Madrid (IVIMA).
} 


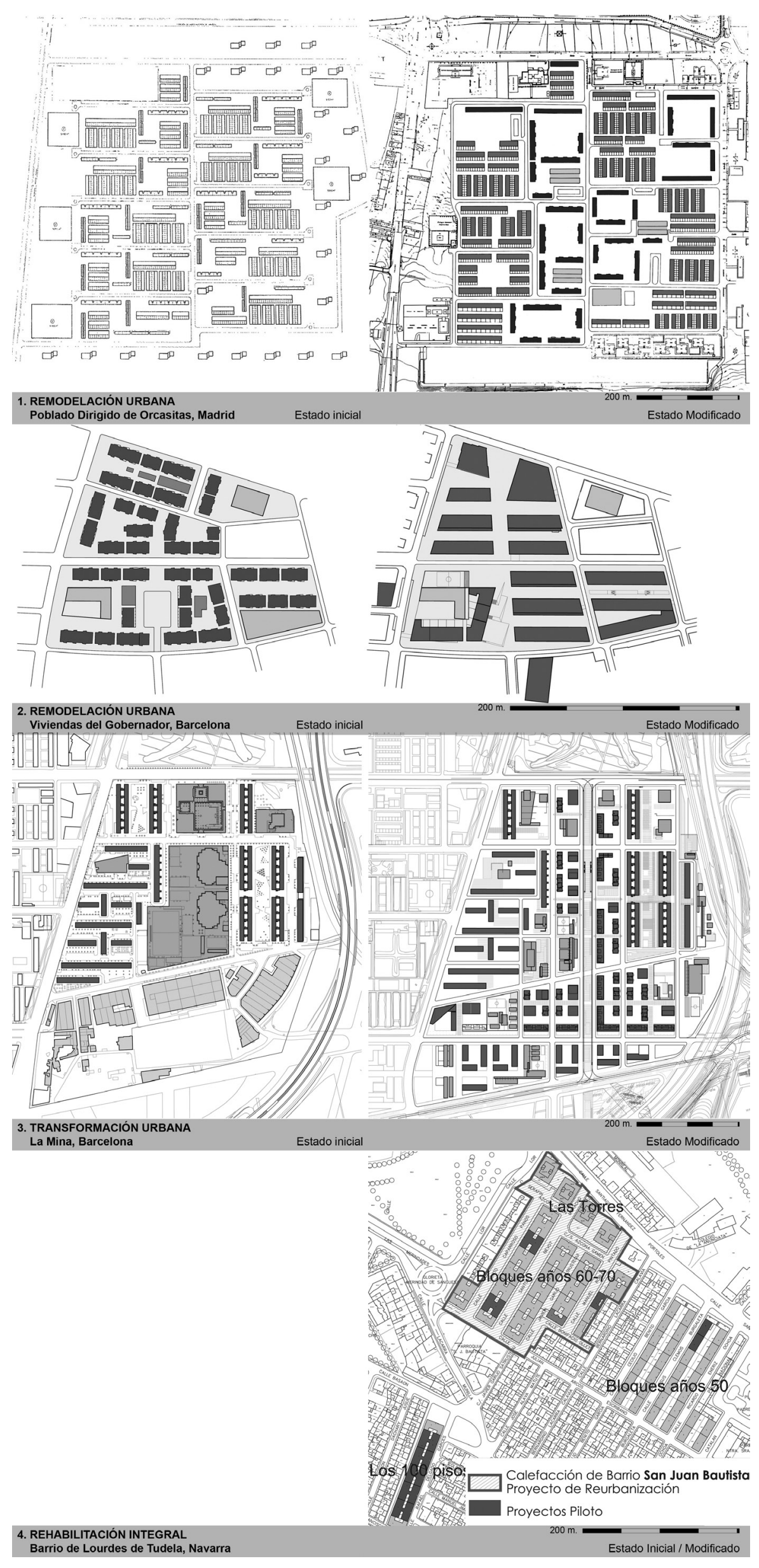

Figura 1. Estructura urbanística de las intervenciones estudiadas. Estado inicial y modificado. 
truida en los años noventa al sur y comercios principalmente el eje central, organizados de forma que no favorecen el tránsito o la actividad.

Los tipos de vivienda se uniformizan, aumentando su superficie construida media de 40 a $105 \mathrm{~m}^{2}$, un $162 \%$ de la superficie inicial, razón por la que se incrementa la altura de los bloques manteniendo el porcentaje de ocupación de suelo (Tabla 1). Esta tipificación de las viviendas no siempre es adecuada para los usuarios, que en algunos casos no pueden mantenerlas, o no llegan a utilizar toda su superficie. Su escasa aportación tipológica y la falta de estrategias adaptadas a la población pueden deberse a varias circunstancias, como son los reducidos márgenes de la ordenanza, los deseos vecinales, la necesidad de acumular edificabilidad o la rapidez con la que se exige la elaboración del proyecto y la ejecución de la obra (4) (Figura 2.1).

La intervención supone una inversión de 102,70 millones de euros (17.090,1 millones de pesetas constantes de 1986), un 25,40 \% del total aportado a los barrios de Promoción Oficial en Madrid hasta que finaliza su ejecución. Al ser pionero en la reivindicación vecinal, se trata de una cantidad muy superior a la del resto, con una media por vivienda de 34.650 euros
(5.764.878 pesetas constantes de 1986) (4). Con ello, se aporta un nuevo alojamiento a la población, que logra su objetivo de quedarse en el barrio, pero la falta de reflexión lleva a una morfología urbana discontinua, que a pesar de la generosidad de recursos económicos, no consigue generar vida (5). La excesiva importancia dada al proceso edificatorio, provoca la escasa atención hacia otras necesidades de sus habitantes y la nula inclusión de usos de carácter productivo, por lo que el polígono, es incapaz de variar su composición social. En cuanto a su relación con el exterior, el empeño de los vecinos de seguir distinguiéndose del entorno, lleva a una operación cerrada en la que los vacíos circundantes no reciben ningún tipo de tratamiento. En definitiva, al considerar la vivienda un fin en sí misma, no se consigue uno de los objetivos de partida del Programa de Remodelación de Barrios, el de crear ciudad, sino que se mantienen los factores que impedían la integración del polígono.

Diferente es el primer caso de remodelación urbana de Barcelona, llevado a cabo en las Viviendas del Gobernador (19922007). Las reivindicaciones vecinales llegan en un momento en el que se asume la situación crítica de más de 8.000 viviendas protegidas, promovidas por el Estado durante la década de los 50. Se pone en marcha una Remodelación de

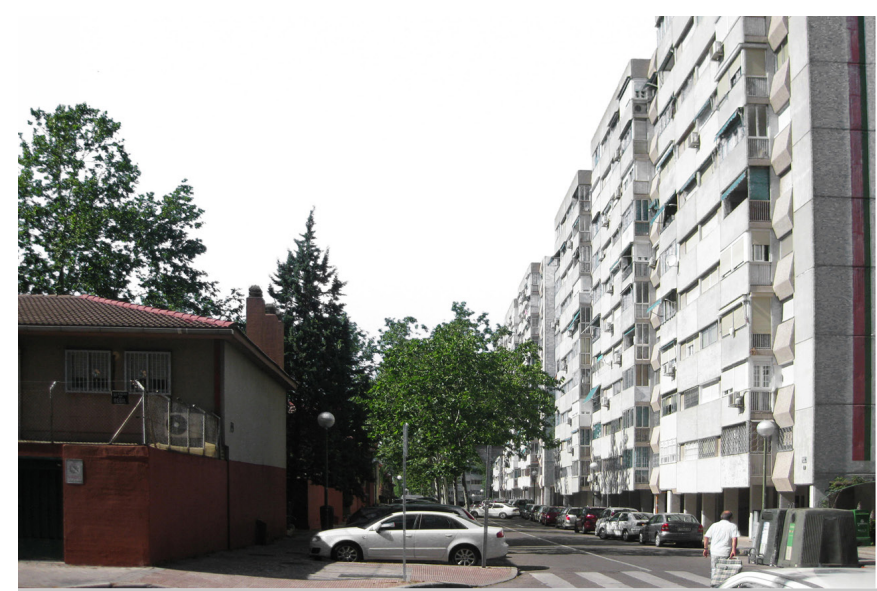

1. REMODELACIÓN URBANA Poblado Dirigido de Orcasitas, Madrid

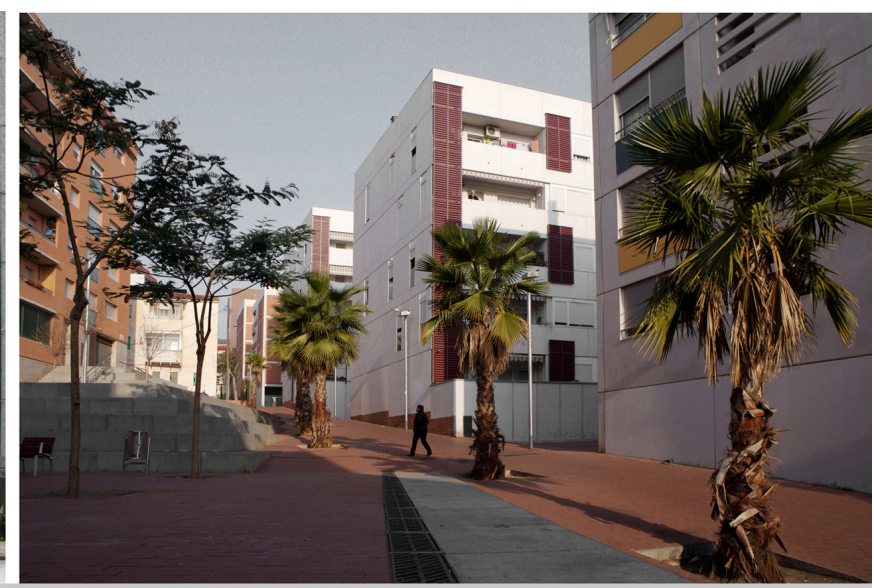

2. REMODELACIÓN URBANA

Viviendas del Gobernador, Barcelona

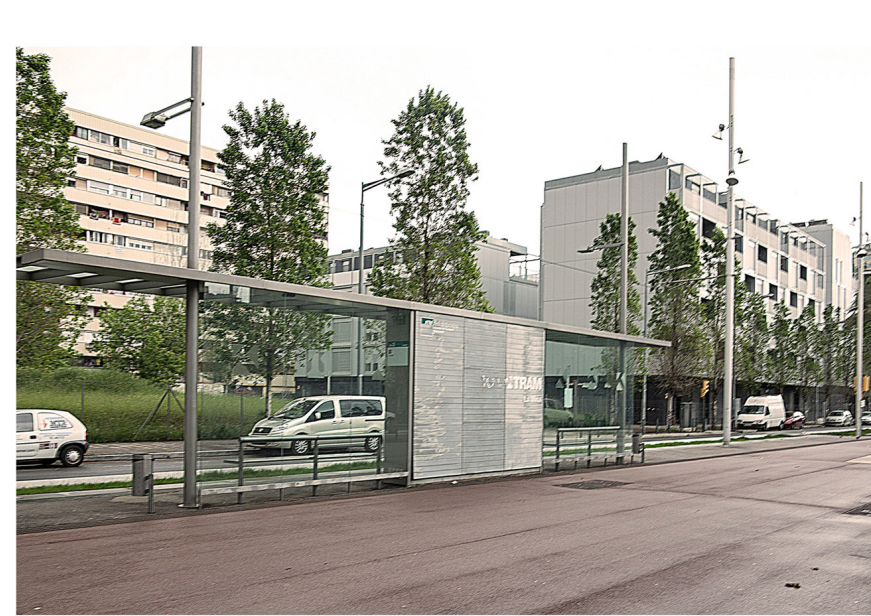

3. TRANSFORMACIÓN URBANA La Mina, Barcelona

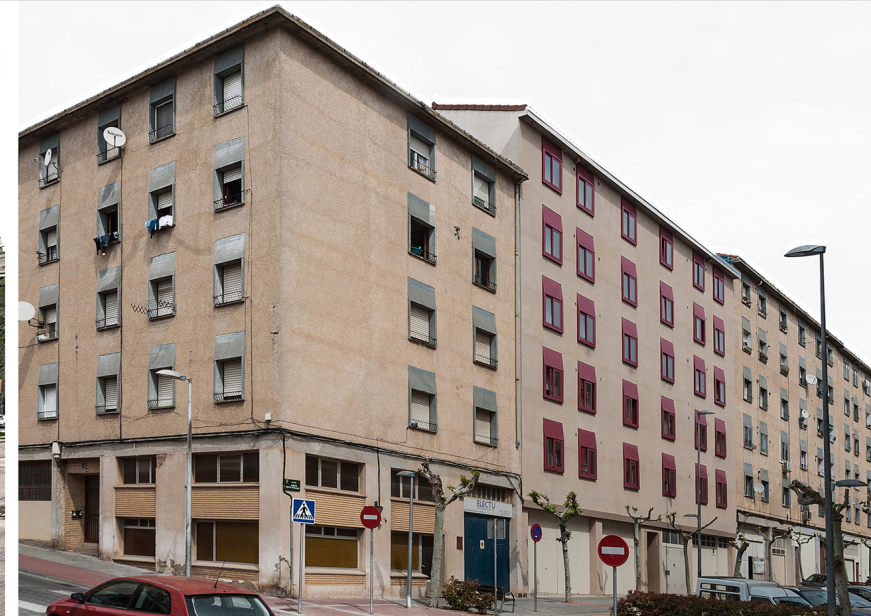

4. REHABILITACIÓN INTEGRAL

Barrio de Lourdes de Tudela, Navarra

Figura 2. Vista actual de los conjuntos residenciales estudiados. 
Barrios principalmente centrada en el área metropolitana de Barcelona, enfocada a la sustitución de vivienda en aquellos conjuntos residenciales en los que la rehabilitación resulta inviable. El encargo de diseñar, planificar, programar y ejecutar las operaciones, asegurando el realojo de la población en el mismo ámbito geográfico, es realizado al Institut Català del Sòl (INCASÒL), como instrumento de la Generalitat de Catalunya.

Las viviendas del Gobernador forman un conjunto de 3,08 ha, construidas en 1952 de forma provisional, con motivo de la celebración del Congreso Eucarístico, para reubicar a los chabolistas afectados por el evento. El proyecto se atribuye a J. Soteras Mauri, y con 906 viviendas y 32 locales comerciales, en su inauguración cuenta con un número aproximado de 5.300 habitantes (6). Su estructura, de carácter rural, se articula alrededor de una plaza, en un juego de terrazas que se adaptan a la pronunciada pendiente. La edificación se organiza en módulos de vivienda corredor de distribución central y tres o excepcionalmente cuatro alturas, unidos por los núcleos de escaleras. La calidad de la construcción es precaria y las condiciones de las viviendas, muy limitadas. Además, el $81 \%$ tiene una superficie construida de $25,00 \mathrm{~m}^{2}$, por lo que se alcanza una densidad altísima, de 294 viviendas por ha, con una ocupación media de seis personas por vivienda. En cuanto a los equipamientos, de todos los servicios previstos en proyecto sólo se llegan a ofrecer los más básicos, resultando insuficientes para toda la población (7).

La ciudad crece rodeando el barrio, pero estableciendo una ruptura que se incrementa en la década de los 80, cuando la grave crisis económica acentúa su degradación. Los espacios públicos, todavía sin pavimentar, se convierten en ámbitos abandonados y residuales, aptos para la marginación y las actividades ilícitas. En cuanto a la situación de las viviendas, los usuarios las van adaptando a su uso, pero su escasa calidad inicial deriva en unas condiciones muy inferiores a cualquier requisito mínimo de habitabilidad. Ante la disyuntiva de intervenir en el barrio realizando labores de mantenimiento, o una remodelación, en 1987 el Patronato Municipal de la Vivienda de Barcelona (PMVB) ${ }^{2}$ encarga un informe al arquitecto J. Vayreda Casadevall para realizar un análisis del estado de la edificación. En él se detalla la debilidad del sistema constructivo, con goteras en el $73 \%$ de las viviendas y humedades de condensación en el $82 \%$. Como conclusión, deja clara la imposibilidad de proceder a una rehabilitación de las viviendas, tanto por sus características constructivas y de conservación, como por la inadecuación de sus dimensiones.

Previo acuerdo político sobre la necesidad de remodelación del barrio, en 1990 quedan delimitadas las funciones y responsabilidades de las distintas administraciones: el Ministerio de Obras Públicas y Urbanismo se compromete a aportar recursos económicos, la Generalitat realizara la gestión y el Ayuntamiento asume la urbanización. El INCASÒL, como encargado de llevar a cabo su ejecución, establece una oficina en el barrio y plantea los objetivos de: derribar todas las edificaciones existentes, construir otras de calidad y tamaño dignos para las familias residentes, recuperar la actividad comercial y adecuar la propuesta al entorno urbano eliminando las fronteras que se habían levantado con los años. La actua- ción responde al Plan Especial de Reforma Interior (PERI) aprobado en 1992, como instrumento urbanístico de referencia en cuanto a decretos y ayudas específicas a los afectados. Es complementado con un censo de residentes que fija en 691 la cantidad de viviendas necesarias, a pesar de las 867 registradas, de las que un $51 \%$ están ocupadas por una o dos personas como máximo.

El proceso de remodelación está condicionado por la carencia de espacio libre, por lo que la primera operación consiste en la rehabilitación de viviendas vacías, previamente adquiridas por el PMVB, para resituar a los vecinos afectados durante las obras, y en elaborar un programa de mantenimiento de edificaciones al límite de su resistencia. La actuación que comienza con una primera demolición en 1993, consigue hasta 2007 completar la construcción de 633 viviendas, 53 locales comerciales, seis locales destinados a equipamientos y 967 plazas de aparcamiento, ubicados en el área de intervención y en suelo externo situado en los límites del polígono. Se reduce así la densidad del barrio a 110,90 viviendas por ha, en un compromiso entre la necesidad de absorber edificabilidad al máximo y de alcanzar una regulación urbanísticamente coherente, mejorando los estándares de viales, aparcamientos, espacios libres y dotaciones comunitarias, tanto en términos cuantitativos como de calidad (Tabla 1).

La adecuación al entorno se plantea desde un punto de vista funcional y de tejido urbano para conseguir un área integrada y permeable conectada con la ciudad existente. Se trata como una plataforma inclinada un $8,70 \%$, articulada en el sentido de la pendiente por un eje peatonal, vertebrador del conjunto y de los espacios libres, que rememora el elemento de reunión de la antigua plaza (Figura 1.2). En la parte alta una torre de diez alturas actúa como hito, en contacto con una vía rápida, y en ambos extremos del eje, edificios de volumetría singular y seis alturas producen modificaciones en la geometría de éste, y se adaptan a la trama existente. El resto de la edificación, en su mayor parte compuesta por bloques de máximo $12 \mathrm{~m}$ de fondo y una media de cinco alturas, se dispone en sentido transversal a la pendiente. Se forman agrupaciones, dos a dos, de manera que delimitan un espacio de carácter estancial y de juego, bajo el cual se sitúa el aparcamiento subterráneo común a ambos. Esta ordenación repetitiva se contrarresta con proyectos diferentes para no generar uniformidad y se organiza, en un sistema más complejo para conseguir la articulación con las calles y edificios adyacentes. En cuanto a las tipologías, se trata de vivienda pasante orientada a cara de mayor soleamiento, en su mayor parte de dos y tres dormitorios y superficie construida media de $77 \mathrm{~m}^{2}$, para dar respuesta a las necesidades de la sociedad, no exclusivamente de los vecinos a realojar (Figura 2.2).

La intervención supone una inversión de 71,68 millones de euros, de la que tres cuartas partes corresponden a las actuaciones de edificación (Tabla 2). La repercusión media por vivienda es de 113.240 euros, un presupuesto abultado en el que se incluyen, el coste de los locales de planta baja, que generan actividad para los nuevos residentes y un excedente de aparcamientos subterráneos, que permiten su uso por parte de los vecinos y compensan su déficit en el entorno. Los espacios destinados a ambos usos, complementarios de la vivien-

\footnotetext{
${ }^{2}$ Entre 1966 y 1967 las viviendas son transferidas al PMVB que realiza la venta a sus usuarios, y se hace cargo de las 59 que quedan sin comprador.para demoler tampoco ha sido derribado por el momento.
} 
da, son vendidos a precio de mercado, generando una serie de beneficios que no han sido computados en la aproximación económica. Por otra parte, esta integración de funciones, unida a la diversidad de piezas edificadas y de espacios públicos, hacen posible que la idea de ciudad esté presente en el resultado, dando lugar a un conjunto que deja de ser reconocible de manera individual y se lleva a la normalidad desde su vocación de apertura.

\section{TRANSFORMACIÓN URBANA}

Dentro de un área pueden desarrollarse operaciones mixtas de remodelación y rehabilitación, que crucen estrategias diferenciadas, adaptándolas a las necesidades de la edificación y de la trama urbana. Un ejemplo de ello es la renovación del barrio barcelonés de La Mina (2000-2010), que supone un proyecto transversal en cuanto a instrumentos y contenidos. El órgano de gestión es el Consorcio del barrio de La Mina, que se crea en el año 2000 para llevar a cabo el Plan de Transformación y supone la base fundamental del compromiso de inversión por parte de la Generalitat de Catalunya, el Ayuntamiento de Sant Adrià de Besòs, el Ayuntamiento de Barcelona y la Diputación de Barcelona ${ }^{3}$.

Situado dentro del término municipal de Sant Adrià de Besòs, el polígono de La Mina, de 26,68 ha, es construido por el PMVB, para realojar a población de bajos recursos procedente de núcleos de autoconstrucción. Según Plan Parcial de 1967, se construye en 1970 bajo la dirección de X. Subias Fages, P. López Iñigo, G. Giráldez Davila y J.A. Ferrater Ducay La Mina Vieja, con 850 viviendas situada en la zona suroeste del barrio. Está formada por bloques exentos, de seis alturas y vivienda pasante, ordenados respecto a dos directrices perpendiculares, que dejan entre sí un sistema de espacios libres de proporciones acotadas. En 1971 una modificación del Plan Parcial da lugar a La Mina Nueva, proyectada por el estudio L35, que está formado por J.I. Galán Martínez, J.L. Martínez Honrubia, J.F. de Mendoza Sans y G. Murtra Ferré. Con 1.850 viviendas, se sitúa al norte y al este de la anterior, asociada a una zona contigua de actividades productivas, conocida como Mina Industrial. La Mina Nueva se construye en un año y es el resultado de una nueva etapa que apuesta por la densidad y la prefabricación. Está constituida por cinco bloques de gran longitud y once alturas, de viviendas con una sola orientación y patios interiores, y por un sexto bloque de ocho plantas y viviendas pasantes. Estos elementos dificultan las relaciones transversales en el interior del barrio y generan espacios libres, de dimensiones desproporcionadas. En la parte central del área, se produce una concentración de grandes equipamientos públicos, que actúan como vacío central entre las dos zonas residenciales, generando una profunda discontinuidad.

Con una población de unos 15.00o habitantes, durante la primera década de existencia del barrio, la marginación toma forma convirtiéndolo en un paradigma de conflicto, de tal forma que llega a los años 90 como un área totalmente estigmatizada. A pesar de que la problemática más importante es de tipo social, la mayor parte de las intervenciones que se llevan a cabo durante este periodo están enfocadas a mejorar la calidad de la edificación. Entre los años 1981 y 1986, el PMVB realiza actuaciones básicas para permitir su cesión al Ayuntamiento de Sant Adrià de Besòs en 1990. Desde este momento y hasta 1992, la Empresa Pla de Besòs, invierte 4,08 millones de euros (680 millones de pesetas constantes de 1992$)^{4}$ en la reparación de instalaciones (42\%), ascensores $(18 \%)$, cerramientos exteriores (21\%) y mantenimiento general (19\%) en acciones aisladas, dirigidas a la restitución del estado inicial de la edificación. Posteriormente, se proponen varios planteamientos que recogen ideas de remodelación y de reducción de densidad. Una de ellas impulsada por el Plan Especial de Ordenación Urbanística del Margen Derecho del Río Besós, propone la reordenación de las zonas central e industrial, incluyendo grandes equipamientos, la rehabilitación con mejora de la edificación de La Mina Vieja y la sustitución de La Mina Nueva por bloques de menor altura. Por primera vez se plantea un proyecto de reestructuración interna, que prima la reactivación económica y social del área desde un punto de vista global (8).

Pero cuando el barrio comienza realmente su transformación es con la combinación de dos circunstancias: la gran operación de finales de los 90, que supone la prolongación de la avenida Diagonal y las actuaciones relacionadas con el Fórum de las Culturas 2004, que resitúan el barrio en el continuo metropolitano (9), y la creación en el año 2000 de un órgano de gestión, capaz de coordinar acciones de política social, financiera y urbanística, como es el Consorcio. La intervención se pone en marcha con un Plan Especial de Reordenación y Mejora (PERM) que se aprueba definitivamente en 2004 y afecta a un sector de 21,21 ha delimitado por el área central de equipamientos, la zona industrial y La Mina Nueva. Para su elaboración se encargan tres estudios desarrollados en 2001: los Estudios de Base Social y Antropológica, dirigidos por la antropóloga C. Doncel Rasillo; el Estudio Técnico sobre los edificios de viviendas, dirigido por el arquitecto C. Díaz Gómez, y los Estudios Urbanísticos de base y alternativas de actuación, redactados por los arquitectos S. Jornet Forner, C. Llop Torné y J.E. Pastor Fernández. En base a ellos se toman dos decisiones:

- En primer lugar, mantener la edificación existente. Teniendo en cuenta el ciclo de mantenimiento llevado a cabo durante los veinte años iniciales, se considera que las labores de rehabilitación ligera con diversas operaciones complementarias son una opción adecuada y económicamente más viable que la rehabilitación integral o la sustitución de la edificación (10).

- En segundo lugar, realizar una densificación de vivienda. A partir del diagnóstico que destaca el escaso espacio ocupado por edificación residencial, en torno al $25 \%$, y la violenta relación entre espacio libre y construido, se considera conveniente una diversificación del barrio que rompa su rigidez.

La nueva ordenación se fundamenta en tres ideas: centralidad, para conferir identidad al conjunto, diversidad para añadir complejidad a nivel físico, social y económico e inter-

\footnotetext{
3 Sus aportaciones se amplían desde el año 2001, con el programa Urban II, financiado por el Fondo Europeo de Desarrollo Regional (FEDER) y con la dotación para intervenciones sociolaborales del Fondo Social Europeo (FSE).para demoler tampoco ha sido derribado por el momento.

${ }^{4}$ Convenio firmado por el MOPU, la Generalitat de Catalunya y el Ayuntamiento de Sant Adrià de Besòs.
} 
cambio para evitar la atrofia y la fractura social (9). Urbanísticamente, se materializa modificando el vacío central con la construcción de un eje urbano condensador de estos tres principios, una rambla sobre la que generar actividad, equipamientos y arquitecturas residenciales (Figura 1.3). Para diseñar el espacio público se trabaja con la distancia y la relación entre las piezas construidas, estableciendo un nuevo sistema de manzanas de $45 \mathrm{~m}$ de ancho y longitud variable entre 60 y $115 \mathrm{~m}$ que acoge un programa diversificado, residencial en su mayor parte. El Plan Especial está basado en una ordenanza flexible en la que se potencia la mezcla tipológica: bloques en dado de $20 \times 20 \mathrm{~m}$, de cuatro a cinco alturas y cuatro viviendas por planta; bloques lineales de anchura máxima de 14 a $15 \mathrm{~m}$ y viviendas pasantes, que dan continuidad a la calle; pequeños bloques de anchura máxima $10 \mathrm{~m}$ perpendiculares a la rambla y piezas singulares de especial significación volumétrica. Se distribuyen además los equipamientos, buscando las mejores localizaciones, para favorecer flujos transversales a la rambla e intercambios del barrio con el resto de la ciudad (Figura 2.3).

La edificación residencial existente se mantiene en su mayor parte, mejorando sus condiciones de habitabilidad y accesibilidad. Se propone también un esponjamiento en La Mina Nueva con la supresión de un edificio y la formación de pasos transversales de tres plantas de altura ${ }^{5}$ en los bloques de mayor longitud. Además, las operaciones realizadas o en curso de ejecución en los edificios de La Mina Nueva son: mejora de la seguridad y del aislamiento de las fachadas; cambio de las cubiertas con incremento del aislamiento térmico; registrabilidad y renovación de las instalaciones existentes; nuevo tramo de escalera desde la última planta, para resolver la evacuación de incendios y operaciones de rehabilitación de ascensores y vestíbulos generales. Esta última actuación consiste en reducir a la mitad, el número de viviendas que acceden por cada portal, inicialmente cada uno daba acceso a dos escaleras y 80 viviendas, lo que conllevaba una gran dificultad respecto a la organización de las comunidades, al mantenimiento y a la convivencia vecinal. Como actividades complementarias, se incluyen la reurbanización y la instalación de ascensores en La Mina Vieja (10).

En el ámbito del Plan Especial, se incrementa el parque residencial hasta completar un total de 3.500 viviendas y una densidad de 165 viviendas por ha, una cifra elevada, que ayuda a conseguir los fines urbanísticos y sociales planteados (Tabla 1). Desde el punto de vista urbanístico, se alcanza una variedad del paisaje urbano, gracias a la diversa morfología de las nuevas piezas, cuya escala contrasta con La
Mina Nueva. La disposición de estos elementos busca generar una serie de transparencias, que permitan percibir los grandes bloques existentes como un telón de fondo desde la rambla. Desde el punto de vista social, se busca la diversidad, al reservar un $80 \%$ de vivienda protegida y un $20 \%$ libre, bajo la idea de obtener un barrio denso y complejo. Por otra parte, el porcentaje de suelo dedicado a equipamientos es muy elevado, incluyendo algunos de mayor significación, como reclamo de población de otros puntos de la ciudad, junto a otros de uso interno del área. Sin embargo la superficie de espacios libres es reducida, debido a la envergadura del elemento más representativo, la rambla y a la presencia de un parque contiguo.

Se trata de una intervención, en la que se busca que la gestión sea factible, con un presupuesto del Consorcio para el periodo 2001-2011 que se eleva a 196,89 millones de euros (Tabla 2) (11). En él se incluyen costes de urbanización, reparcelación, rehabilitación, nuevas viviendas protegidas y programas sociales y supone una repercusión media para cada una de las viviendas protegidas de 70.320 euros. Esta cifra resulta más reducida que la de un proceso de remodelación, debido a que supone una media entre la construcción de edificios de nueva planta, con grandes inversiones en periodos relativamente cortos, y la mejora las deficiencias más notables de los edificios existentes, en una actuación más prolongada en el tiempo.

\section{REHABILITACIÓN INTEGRAL}

Las rehabilitaciones integrales se plantean como medidas conservativas, que atienden fundamentalmente al valor de conjunto de los grupos edificatorios y están enfocadas a mejorar su habitabilidad y su integración en el entorno cercano. Este tipo de actuación se está llevando a cabo en varios grupos de vivienda protegida construidos en Zaragoza, entre las décadas de los años 40 y 60. Se trata de conjuntos residenciales, que desde el punto de vista constructivo, presentan carencias de tipo funcional, denotando dificultades a otros niveles: económico, social o cultural. La Administración se implica yendo más allá de la mera regulación para aplicar políticas de recuperación en estas áreas de mayor vulnerabilidad. En este caso, 21 de estos grupos residenciales son catalogados como Conjuntos Urbanos de Interés el año 2001, como medida para controlar su conservación. Posteriormente, en 2004, el Ayuntamiento, a través de la Sociedad Municipal Zaragoza Vivienda, sienta las bases metodológicas para estudiar su realidad física y social, y llevar a cabo una intervención urbana a largo plazo con criterios de innovación, conexión y sostenibilidad medioambiental (12). Con ello, genera un mo-

Tabla 1. Datos urbanísticos básicos de los conjuntos residenciales tras las intervenciones.

\begin{tabular}{|l|c|c|c|c|}
\hline & $\begin{array}{c}\text { Edificación } \\
\text { Residencial }\end{array}$ & $\begin{array}{c}\text { Edificación } \\
\text { Otros usos }\end{array}$ & Zonas libres & $\begin{array}{c}\text { Viario y } \\
\text { aparcamiento }\end{array}$ \\
\hline P.D. Orcasitas & $16,37 \%$ & $8,98 \%$ & $46,56 \%$ & $30,16 \%$ \\
\hline Viviendas del Gobernador & $41,00 \%$ & $10,00 \%$ & $15,60 \%$ & $33,40 \%$ \\
\hline La Mina & $35,60 \%$ & $17,31 \%$ & $19,16 \%$ & $27,93 \%$ \\
\hline Barrio de Lourdes & $45,00 \%$ & $0,00 \%$ & $7,60 \%$ & $47,40 \%$ \\
\hline
\end{tabular}

\footnotetext{
${ }^{5}$ Su estructura de encofrado túnel permite quitar tres módulos sin que se resienta el resto de la edificación. Se ha barajado también la posibilidad de formar un corte de unos $15 \mathrm{~m}$ en cada toda su altura, pero aún no se ha realizado ninguna de las dos soluciones. El bloque propuesto para demoler tampoco ha sido derribado por el momento.
} 
Tabla 2. Desglose de importes de las actuaciones desarrolladas en los tres tipos de intervención.

Actuaciones desarrolladas entre 1992 y 2007 en la Remodelación de las Viviendas del Gobernador:

\begin{tabular}{|l|c|c|}
\hline & $\begin{array}{c}\text { Ejecutado importe } \\
\text { (millones } \mathbf{~ ) ~}\end{array}$ & $\begin{array}{c}\text { Ejecutado } \\
\text { (\%) }\end{array}$ \\
\hline Expropiaciones & 9,56 & 13,35 \\
\hline Derribo y obras de mantenimiento & 4,10 & 5,70 \\
\hline Urbanización espacios públicos y conectividad & 2,82 & 3,95 \\
\hline Edificación & 55,20 & 77,00 \\
\hline Total & 71,68 & 100,00 \\
\hline
\end{tabular}

Actuaciones desarrolladas entre 2001 y 2011 en el Plan de Transformación de La Mina:

\begin{tabular}{|l|c|c|}
\hline & $\begin{array}{c}\text { Ejecutado importe } \\
\text { (Millones€) }\end{array}$ & $\begin{array}{c}\text { Ejecutado } \\
\text { (\%) }\end{array}$ \\
\hline Social & 29,20 & 14,80 \\
\hline Equipamientos & 22,40 & 11,40 \\
\hline Mejora accesibilidad y rehabilitación viviendas & 10,30 & 5,20 \\
\hline Urbanización espacios públicos y conectividad & 26,56 & 13,50 \\
\hline Nuevas viviendas protegidas y derribos afectados & 45,63 & 23,20 \\
\hline Proyecto de reparcelación & 31,01 & 15,80 \\
\hline Valor de suelo cedido por las administraciones & 22,59 & 11,50 \\
\hline Estructura & 7,74 & 3,90 \\
\hline Provisión financiera & 1,46 & 0,70 \\
\hline Total & 196,89 & 100,00 \\
\hline
\end{tabular}

Actuaciones desarrolladas entre 2010 y 2011 en la Rehabilitación del Barrio de Lourdes:

\begin{tabular}{|l|c|c|}
\hline & $\begin{array}{c}\text { Ejecutado importe } \\
\text { (Millone s€) }\end{array}$ & $\begin{array}{c}\text { Ejecutado } \\
\text { (\%) }\end{array}$ \\
\hline Bloques 50 & 0,62 & \\
\hline Los 100 Pisos & 1,85 & \\
\hline Bloques 60-70 & 0,95 & 45,60 \\
\hline Total proyectos piloto & 3,07 & 41,00 \\
\hline Renovación district heating & 2,76 & 13,30 \\
\hline Reurbanización & 0,91 & 100,00 \\
\hline Total & 6,73 & \\
\hline
\end{tabular}

delo eficaz, que técnicamente se plantea como una actuación masiva y sistemática, tomando como exigencia básica la normativa vigente, y socialmente, como un proceso de información-participación.

En uno de los proyectos piloto, la rehabilitación de un bloque de 40 viviendas en el Grupo Girón, con proyecto original de A. Allanegui Félez y F. García Marco, realizada por G. Molpeceres López y M. Abad Lavilla en 2007, se llevan a cabo las siguientes actuaciones: mejora del comportamiento higrotérmico de la envolvente (48,50\%); actualización de las instalaciones (29,90\%); accesibilidad e instalación de ascensores (19,00\%), y labores mínimas de urbanización (2,60\%). La optimización de instalaciones y el revestimiento de fachadas como medidas pasivas, complementadas con la incorporación de captadores solares en cubierta, consiguen incrementar el grado de confort y de eficiencia, reduciendo el consumo anual medio por vivienda hasta en un $49 \%$. Esto hace que la intervención adquiera un gran valor medioambiental, tanto por el propio concepto del proceso, como por el ahorro de emisiones a lo largo del nuevo ciclo de vida de la edificación. El presupuesto de este proyecto tipo, asciende a un total de 1.775.650 euros, lo que supone un coste medio de inversión por vivienda de 44.400 euros, con una aportación pública para este primer momento del proceso del $76 \%$.

Este modelo de intervención, se aplica años más tarde en el proyecto Lourdes Renove en Tudela, Navarra (2010-2011). Forma parte del Proyecto Eco-City, cofinanciado por el Programa Europeo Concerto, iniciativa que apoya a las comunidades locales en la reducción de emisiones de $\mathrm{CO}_{2}$. El Ayuntamiento de Tudela lo concibe como la primera fase de un Proyecto Global de Rehabilitación Energética Integral del Barrio de Lourdes, con el que marca las pautas de calidad para la actuación completa. La coordinación de la operación corre a cargo de un equipo de gestión, la empresa pública del Gobierno de Navarra Nasuvinsa, que controla los aspectos técnicos, económicos, financieros, urbanísticos, jurídicos y sociales. Su conocimiento del proceso llevado a cabo en Zaragoza, le permite hacerlo suyo y optimizarlo, teniendo un mayor control sobre el proyecto, sobre el resultado y sobre el plazo de ejecución.

El barrio de Lourdes, de 13,00 ha es un distrito de Vivienda Social, promovido entre 1954 y 1972, con una identidad marcada en el continuo urbano de Tudela. Contiene un grupo 
de viviendas unifamiliares más 768 pisos que pertenecen a varios conjuntos edificatorios concatenados. El primero, proyectado por D. Ariz Armendáriz, contiene unos bloques de los años 50, de tres alturas con 176 viviendas. El resto, proyectados por J. Moya Arderius, están formados por: un bloque llamado Los 100 Pisos, de cinco alturas con 106 viviendas; bloques de las décadas de los 60 y 70 , de cinco alturas con 374 viviendas; y torres de ocho alturas con 112 viviendas. Las débiles condiciones constructivas de los edificios, sus problemas de accesibilidad y el deterioro de las instalaciones, alejan a este barrio de la demanda inmobiliaria, lo que motiva una pérdida de residentes, constituidos en su mayor parte por la envejecida población original y por nuevos habitantes de origen extranjero. A pesar de la dificultad que supone trabajar con una población tan vulnerable y más en una situación de crisis económica, el Ayuntamiento decide actuar para frenar este proceso de deterioro físico y social. Consciente de la importancia de la participación ciudadana, desde el inicio busca la colaboración de los vecinos, representados por las Comunidades de Propietarios, que van a ser las figuras con las que se tramita el proceso y a las que se aporta financiación.

En marzo de 2010, Nasuvinsa abre una oficina de barrio, que actúa como punto de encuentro entre administración, técnicos y vecinos, y en septiembre del mismo año, se aprueba una Ordenanza municipal específica, que regula las ayudas a la elaboración de las obras de rehabilitación de instalaciones comunes y vivienda colectiva, en el ámbito del proyecto. Para esta primera fase se incluyen las cuatro tipologías edificatorias de vivienda colectiva sobre las que se desarrollan tres Proyectos en paralelo: Reurbanización, Renovación del District Heating o Sistema de Calefacción de barrio, incluyendo el uso de energías renovables y Rehabilitación Integral de la edificación (Figura 1.4).

Los Proyectos de Reurbanización y de Renovación del District Heating, se llevan a cabo en un ámbito de 4,45 ha, que abarca las tipologías de bloque de los años del 6o al 70, y de torre, con un total de 486 viviendas. El Proyecto de Reurbanización es redactado por el arquitecto M. Blasco Esparza y consiste en la recuperación de espacio para el peatón, con inclusión de zonas verdes y mejora de la accesibilidad. El Proyecto de Renovación del District Heating, existente en esta área del barrio, viene motivado por el bajo rendimiento del sistema de producción y por el deterioro de la red de distribución, en la que se producen fugas y falta de estanqueidad. Se recurre a una renovación integral de la instalación, como medida que garantice un adecuado funcionamiento. Se construyen nuevas salas de calderas de biomasa y gas $^{6}$, de tal forma que las de biomasa funcionan para satisfacer la demanda de calor base, produciendo un $80 \%$ del total de la energía, y las de condensación de gas, las complementan para cubrir puntas de demanda. Se renuevan también las redes de distribución, tanto generales hasta los portales, como individuales aportando nuevos montantes, contadores, termostatos y circuitos, para garantizar una temperatura adecuada en las viviendas.

La Rehabilitación Integral de la edificación se ejecuta en tres proyectos piloto para 146 viviendas, pertenecientes a las tipologías en bloque construidas en los años 50, en los años del 60 al 70, y en Los 100 Pisos. Arranca con un diagnóstico previo de la edificación desarrollado por los técnicos de Nasuvinsa, que les da un conocimiento de la situación y les sirve para elaborar los pliegos de una serie de concursos de ideas convocados en junio de 2010, con intervención de jurado. En ellos se pide que cada propuesta vaya acompañada de una valoración económica desglosada por actuaciones y ratificada por una empresa constructora con experiencia en el sector, para aportar desde este primer momento costes aproximados a las Comunidades de Propietarios. De esta forma se reducen los tiempos, se selecciona un modelo de rehabilitación específico para cada tipología y se garantiza la viabilidad de cada solución. Los proyectos ganadores que se terminan ejecutando son: el de LKS Ingeniería para los bloques de los años 50, donde se actúa en dos portales (12 viviendas); el del equipo de Margallo y Orgambide arquitectos para Los 100 pisos, donde se acometen cinco portales (90 viviendas) y el del estudio MAR arquitectos liderado por G. Molpeceres López, para los bloques de los años del 60 al 70 , donde se interviene en tres portales (44 viviendas).

Como requisito básico, en cada intervención se exige la posibilidad de llevar a cabo la obra sin realojar a la población y la incorporación de medidas de eficiencia energética, para alcanzar no solo las condiciones recogidas en el CTE, sino las específicas del Proyecto Eco-City. Se aportan por ello soluciones integrales, para la mejora del comportamiento higrotérmico de la envolvente actuando en cubiertas, bajo las viviendas situadas en la planta inferior y fachadas. Estas últimas se solucionan con sistema $S A T E$ y aportan una composición estética adecuada a cada tipología, que se complementa con la duplicación de carpintería por el exterior y la superposición de elementos que mejoran su funcionamiento. Se renuevan también las instalaciones generales hasta cada vivienda y se eliminan barreras arquitectónicas actuando en portales y añadiendo ascensores cuando existen más de tres alturas (Figura 2.4).

La inversión total alcanza los 6,73 millones de euros (Tabla 2) de los que llama la atención el alto porcentaje que supone la renovación del district heating, que tiene una repercusión por vivienda de 5.670 euros. Gracias a la monitorización de la instalación, se ha podido comprobar que la renovación integral del district heating con funcionamiento del sistema las 24 horas, supone una reducción de consumo de un $48 \%$, siendo el ahorro de gas de un $89 \%$. En las viviendas rehabilitadas, las medidas pasivas permiten un ahorro de consumo de un $45 \%$ de media, con un aumento considerable de su confort térmico. En los casos en los que se efectúan ambas actuaciones, con una inversión media por vivienda de 29.220 euros y aportación pública del $60 \%$, el consumo se reduce por encima del $70 \%$ de media. Estos datos subrayan la importancia de la rehabilitación energética aplicada a nivel de barrio, no solo como herramienta para combatir su degradación, sino también la falta de eficiencia de un parque edificatorio obsoleto.

\section{DISCUSIÓN Y CONCLUSIONES}

De la investigación y el análisis realizados se han extraído las siguientes conclusiones:

1. Intervenir en este tipo de promociones es rentable, por la mejora que supone en cuanto a habitabilidad, ahorro

\footnotetext{
${ }^{6}$ Dos calderas de biomasa VIESSMANN con potencia nominal $720 \mathrm{~kW}$ y agua a $85 / 65^{\circ} \mathrm{C}$ y tres calderas de condensación HOVAL con potencia nominal $665 \mathrm{~kW} \mathrm{a} 80 / 60^{\circ} \mathrm{C}$. La potencia total instalada es de $3.365 \mathrm{~kW}$.
} 
Tabla 3. Comparativa de inversiones entre las distintas intervenciones.

\begin{tabular}{|l|c|c|c|c|}
\hline & $\begin{array}{c}\text { Remodelación } \\
\text { P.D. Orcasitas } \\
\text { (Madrid) }\end{array}$ & $\begin{array}{c}\text { Remodelación } \\
\text { Viv. Gobernador } \\
\text { (Barcelona) }\end{array}$ & $\begin{array}{c}\text { P. Transformación } \\
\text { La Mina } \\
\text { (Barcelona) }\end{array}$ & $\begin{array}{c}\text { Rehabilitación } \\
\text { Barrio Lourdes } \\
\text { (Tudela) }\end{array}$ \\
\hline Superficie (Ha) & 40,27 & 3,08 & 21,21 & 4,45 \\
\hline Viviendas total & 2.964 & 906 & 1.850 & 486 \\
\hline Viviendas interv. & 2.964 & 633 & 2.800 & 44 \\
\hline Año & 1.986 & 2.007 & 2.010 & 2.011 \\
\hline Inversión total & 102,70 & 71,68 & 196,89 & 3,67 \\
(millones $€$ ) & $271,74^{*}$ & $82,00^{*}$ & $212,69^{*}$ & $3,82^{*}$ \\
\hline $\begin{array}{l}\text { Repercusión } \\
\text { por vivienda }(€)\end{array}$ & 34.650 & 113.240 & 70.320 & 29.220 \\
\hline
\end{tabular}

* $€$ constantes año 2014. Actualizado con el IPC general (sistema IPC base 2011) para periodos anuales completos del Instituto Nacional de Estadística.

energético, cohesión socioeconómica y conectividad urbanística con la ciudad, consiguiendo eliminar factores de discriminación social como la pobreza energética y la marginalidad.

Para que el proceso sea exitoso, debe haber una corresponsabilidad económica entre los vecinos y la administración, sin que todo el peso recaiga sobre ésta. Para ello han de buscarse vías de gestión con el sector privado, como la productividad de rendimientos energéticos, incrementos en el aprovechamiento de suelo o la incorporación de nuevos usos, que hagan posibles modelos de gestión sostenibles.

2. Comparando los tres tipos de intervención, se observa que la rehabilitación, es el sistema económicamente más viable (Tabla 3) y el que por tanto puede tener un mayor alcance, debido a las cantidades totales que maneja y a su posibilidad de desarrollo en periodos de tiempo largos, y con una afección mínima a la población. Pero debe acometerse cuando los indicadores, no sólo físicos, sino también sociales lo recomienden y no apunten a actuaciones más profundas, que en cualquier caso solo serán aplicadas a escala de barrio, como viene manifestándose a lo largo de todo el estudio.

Se observa también que aunque en un proceso de transformación urbana la inversión económica sea más cuantiosa que en uno de remodelación, la repercusión por vivienda es menor. Hay que tener en cuenta que en las cifras de la Tabla 3 , la inversión total y la repercusión por vivienda incluyen precio del suelo, derribos, urbanización, instalaciones de barrio, construcción de viviendas, aparcamientos, comercio y equipamientos públicos. Esto conduce a que intervenciones con grandes dotaciones públicas, como la de Orcasitas, tengan una repercusión más reducida que otras, como la de Viviendas del Gobernador, en las que la operación sirve para corregir carencias del área tratada y de su entorno.

3. Para facilitar el éxito de la intervención es imprescindible la participación de los vecinos, matizándola y ponderándola, con un tipo de gestión como el que se viene realizando en las operaciones más actuales. Queda así el caso de Orcasitas, como un proceso pionero, excepcional, por el momento histórico y la implicación de sus habitantes, y totalmente experimental.

4. En estas claves ha de enmarcarse la definición de las políticas de intervención para estos potenciales espacios de exclusión, donde a deficiencias de orden constructivo, urbanístico y conceptual, se unen otras de tipo socioeconómico. Por tanto, se tiende a conseguir su recuperación, afrontando la problemática que los rodea de manera global.

\section{AGRADECIMIENTOS}

Fuentes: Archivo Regional de la Comunidad de Madrid; Archivo del IVIMA, Madrid; INCASOL, Barcelona; Jornet Llop Pastor Arquitectos, Barcelona y Nasuvinsa, Pamplona.

Este trabajo se ha hecho en el marco del proyecto de investigación «Protocolo de Diseño Integrado para la Rehabilitación de la Vivienda Social y Regeneración Urbana» (BIA201344001-R) dentro del Programa Estatal de I+D+i orientada a los restos de la sociedad, financiado por el Ministerio de Economía y Competitividad.

\section{REFERENCIAS}

(1) Castro, P., Molina, J. (1996). Un ejemplo de participación y renovación urbana: la remodelación de barrios en Madrid (España). Ciudades para un Futuro más Sostenible. http://habitat.aq.upm.es/dubai/96/bp258.html.

(2) Leoz, R., Ruiz, J. (1961). El poblado de Orcasitas (cont.). TA. Temas de Arquitectura y Urbanismo, 27: $922-926$.

(3) Moya, L. (Coordinador) Barrios de Promoción Oficial de la Operación de Remodelación. Madrid 1979-1986. Madrid: Instituto de la Vivienda de Madrid (IVIMA), Conserjería de Ordenación del Territorio, Medio Ambiente y Vivienda Comunidad de Madrid.

(4) Moya, L. (1987). Análisis del Proceso de Remodelación y Realojamiento de Barrios en Madrid. Madrid: Escuela Técnica Superior de Arquitectura de Madrid (ETSAM)/ Instituto de la Vivienda de Madrid (IVIMA).

(5) Rodríguez-Villasante, T., Alguacil, K., Denche, C., Hernández Aja, A., León, C., Velázquez, I. (2009). Retrato de chabolista con piso. Análisis de redes sociales en la remodelación de barrios de Madrid. Madrid: ALFOZ-CIDUR/ IVIMA-SGV.

(6) Donato, J.E. (1965). Barrios altos de San Andrés. Cuadernos de arquitectura, 60: 19-40.

(7) Pradas, R. (2008). Vivendes del Governador. Una historia urbana. Barcelona: Institut Català del Sòl.

(8) Blos, D. (1999). Los polígonos de vivienda social: Perspectivas hacia su recuperación en España, Francia y Brasil. Tesis doctoral no publicada de la Universidad Politécnica de Cataluña. Barcelona: Escuela Técnica Superior de Arquitectura de Barcelona (ETSAB). 
(9) López, R. (2009). Ordenar el territorio, proyectar la ciudad, rehabilitar los tejidos existentes. La relevancia del planeamiento a través de los premios nacionales de urbanismo. Madrid: Ministerio de la Vivienda.

(10) Díaz, C., Conrado, C., Llorens, I., Pardo, F., Hormías, E. (2012). Un estudio de caso: la rehabilitación de los edificios de viviendas del barrio de La Mina en Sant Adrià del Besòs (Barcelona). Análisis funcional y de las condiciones de seguridad, habitabilidad y mantenimiento. Informes de la Construcción, 64(525): 19-34, doi: http://dx.doi.org/10.3989/ ic.11.005.

(11) Barrio de la Mina. El Plan de Transformación. (2007). http://www.barrimina.cat/cast/index.php/plan-de-transformaciainmenu-39.

(12) Ruiz, L.G., Rubio, J. (2006). Nuevas propuestas de Rehabilitación Urbana en Zaragoza Estudio de Conjuntos Urbanos de Interés. Zaragoza: Sociedad Municipal de Rehabilitación Urbana de Zaragoza (SMRUZ). 